\title{
Shift from Goal-Directed to Habitual Cocaine Seeking after Prolonged Experience in Rats
}

\author{
Agustin Zapata, Vicki L. Minney, and Toni S. Shippenberg \\ Integrative Neuroscience Section, National Institutes of Health, National Institute on Drug Abuse Intramural Research Program, Baltimore, \\ Maryland 21224
}

The development of drug-seeking habits is implicated in the transition from recreational drug use to addiction. Using a drug seeking/taking chained schedule of intravenous cocaine self-administration and reward devaluation methods in rats, the present studies examined whether drug seeking that is initially goal-directed becomes habitual after prolonged drug seeking and taking. Devaluation of the outcome of the drug seeking link (i.e., the drug taking link of the chained schedule) by extinction significantly decreased drug seeking indicating that behavior is goal-directed rather than habitual. With, however, more prolonged drug experience, animals transitioned to habitual cocaine seeking. Thus, in these animals, cocaine seeking was insensitive to outcome devaluation. Moreover, when the dorsolateral striatum, an area implicated in habit learning, was transiently inactivated, outcome devaluation was effective in decreasing drug seeking indicating that responding was no longer habitual but had reverted to control by the goal-directed system. These studies provide direct evidence that cocaine seeking becomes habitual with prolonged drug experience and describe a rodent model with which to study the neural mechanisms underlying the transition from goal-directed to habitual drug seeking.

\section{Introduction}

Studies of instrumental conditioning in rats have shown that two distinct processes control actions that are instrumental to gaining access to rewarding stimuli. During early learning, behavior is goal-directed, dependent on action-outcome associations and performance is readily influenced by changes in outcome value. As training proceeds, control over performance shifts to a stimulus-response process. Actions become habitual, and insensitive to changes in instrumental contingency or reward value (Adams and Dickinson, 1981; Dickinson, 1985; Balleine and Dickinson, 1998). As a consequence, assessment of changes in operant responding after outcome devaluation is used to differentiate goal-directed from habit-driven behaviors (Adams and Dickinson, 1981; Colwill and Rescorla, 1985; Dickinson, 1985).

Habit learning processes have been implicated in the transition from recreational drug use to the compulsive drug seeking that characterizes addiction (White, 1996; Everitt and Robbins, 2005; Everitt et al., 2008). Lesions of the dorsolateral striatum, an area necessary for habit learning (Packard and Knowlton, 2002; Yin et al., 2004), attenuate cue controlled drug seeking (Ito et al., 2002; Vanderschuren et al., 2005; Fuchs et al., 2006; Belin and Everitt, 2008) and nigrostriatal dopamine lesions disrupt habit

\footnotetext{
Received Aug. 4, 2010; revised Sept. 14, 2010; accepted Sept. 20, 2010.

This work was supported by funding from the Intramural Research Program, National Institute on Drug Abuse, National Institutes of Health, Department of Health and Human Services.

Correspondence should be addressed to Dr. Agustin Zapata, Integrative Neuroscience Section, National Institute on Drug Abuse Intramural Research Program, 333 Cassell Drive, Baltimore, MD 21224. E-mail: Azapata@ mail.nih.gov.

DOI:10.1523/JNEUROSCI.4072-10.2010

Copyright $\odot 2010$ the authors $\quad 0270-6474 / 10 / 3015457-07 \$ 15.00 / 0$
}

formation (Faure et al., 2005). Moreover, prior repeated psychostimulant administration facilitates habitual responding for food reinforcers (Schoenbaum and Setlow, 2005; Nelson and Killcross, 2006; Nordquist et al., 2007) suggesting that cocaine administration accelerates the development of habits.

Studies assessing the development of habitual drug seeking are limited. Using satiation or pairing of oral reinforcers with $\mathrm{LiCl}$ induced sickness, two studies have demonstrated habitual drug seeking of orally administered drug reinforcers (Dickinson et al., 2002; Miles et al., 2003). However, studies of intravenous drug administration have been hampered by difficulties inherent in adapting standard devaluation procedures for natural reinforcers to those of intravenously administered drug. In contrast to natural reinforcers, this route of administration is not associated with an obvious consummatory response and psychostimulants such as cocaine have unconditioned, behaviorally activating, effects that can affect responding.

In an elegant series of studies, Olmstead et al. (2001) circumvented these issues by use of a heterogeneous chained schedule of intravenous cocaine administration in which habitual cocaine seeking was tested by devaluing the final link of a drug seeking/ taking chained schedule. In this procedure, responding on the designated drug seeking lever provides access to a drug taking lever, rather than to cocaine itself. Responses on the drug taking lever result in a cocaine infusion. Devaluation of the drug taking link (by extinction) was assessed once performance under the chained schedule had stabilized. Decreased responding during the drug seeking link was observed indicating that behavior was goal-directed, rather than habitual. Fundamental questions, however, remain as to whether extended training on the chained schedule produce habitual drug-seeking responses that are insen- 
sitive to devaluation. Such information is important for current theories of addiction and for identifying the contingencies that control drug self-administration.

Accordingly, in the present studies we have used an adaptation of the seeking/taking chained schedule of Olmstead et al. (2001) to address this issue. Given the documented role of the dorsolateral striatum in stimulus-response associations (Yin et al., 2004), the influence of inactivation of this brain area was assessed in animals with a prolonged history of cocaine seeking and taking.

\section{Materials and Methods}

\section{Subjects}

Male Long-Evans rats (Charles River Laboratories) weighing $300 \mathrm{~g}$ at the beginning of experiments were housed $2-3$ per cage for at least 1 week before use in facilities accredited by the American Association for the Accreditation of Laboratory Animal Care. They were maintained in a temperature- and humidity-controlled environment under a reverse $12 \mathrm{~h}$ light/dark cycle with food and water available ad libitum. One week after surgery, food was restricted to $15 \mathrm{~g}$ of standard rodent diet per day and made available after the daily self-administration sessions. Experiments were conducted during the dark cycle. All experiments were approved by the Institutional Care and Use Committee of the National Institute on Drug Abuse (NIDA), National Institutes of Health (NIH) (Rockville, MD) and conducted in accordance with the Guide for the Care and Use of Laboratory Animals provided by the NIH and adopted by the NIDA Intramural Research Program.

\section{Surgery}

Rats were anesthetized with equithesin ( $1 \%$ pentobarbital, $2 \%$ magnesium sulfate, $4 \%$ chloral hydrate, $42 \%$ propylene glycol, $11 \%$ ethanol, 3 $\mathrm{ml} / \mathrm{kg}$, i.p.) and a SILASTIC catheter (inner diameter, 0.020 inch; outer diameter, 0.037 inch; Dow Corning) was advanced $3.5 \mathrm{~cm}$ into the right jugular vein. The catheter terminated in an L-shaped steel tube mounted on top of the skull with cranioplastic cement and was secured with 3 stainless steel screws. Catheters were flushed daily with $0.1 \mathrm{ml}$ of heparinized saline to maintain patency. Animals were allowed to recover for at least 1 week after the surgery and before food restriction commenced. Catheters were flushed once daily $(0.1 \mathrm{ml})$ with $25 \mathrm{mg} / \mathrm{ml}$ gentamicin for $5 \mathrm{~d}$ after surgery and then with $120 \mathrm{mg} / \mathrm{ml}$ cefazolin throughout the study. Operant training started 2-3 d after the beginning of food deprivation.

Subjects in experiment 3 were stereotaxically implanted with bilateral guide cannulae (C315, Plastics One) aimed $1 \mathrm{~mm}$ dorsal to the dorsolateral striatum (anteroposterior, +0.5 ; lateral, \pm 3.6 ; ventral, $-3.6 \mathrm{~mm}$ relative to bregma).

\section{Behavioral procedures}

Experiment 1. Operant training took place in rat operant chambers equipped with 2 retractable levers (Med Associates). The training protocol was adapted from Olmstead et al. (2001). Animals were trained to lever press one lever (designated as the drug taking lever) for intravenous cocaine infusions under a fixed ratio 1 (FR1) schedule. Each lever press resulted in infusion of $0.75 \mathrm{mg} / \mathrm{kg}$ cocaine accompanied by retraction of the taking lever, extinction of the house light and illumination of a cue light above the taking lever for $30 \mathrm{~s}$. Animals were allowed to selfadminister for a maximum of 40 infusions or $2 \mathrm{~h}$. After reliable selfadministration was established (2 consecutive sessions $>10$ infusions/ session), simultaneous presentation of sucrose ( $10 \mu \mathrm{l}$ of $20 \%$ sucrose via a liquid dipper) was initiated. The dipper cup containing sucrose, which was located in the food magazine, remained in the up position. The first head entry into the dipper magazine after a random interval $60 \mathrm{~s}$ (RI60) schedule had elapsed resulted in refilling of the sucrose cup. Head entries into the dipper magazine were recorded as a measure of a Pavlovian approach response (Corbit and Balleine, 2005) and used to control for nonspecific effects on general activity. Cocaine self-administration under the FR1 (fixed ratio 1) schedule continued with simultaneous access to sucrose under a RI60 schedule until performance stabilized (3 consecu- tive sessions $>20$ infusions and $<20 \%$ between-session variation). A chained schedule was then introduced for cocaine self-administration. Every infusion cycle started with the insertion of a second lever, designated as the drug seeking lever. The first lever press on the seeking lever after completion of an RI2 s schedule resulted in retraction of the seeking lever and extension of the taking lever. The first response on the taking lever resulted in the cocaine infusion sequence (e.g., infusion of 0.75 $\mathrm{mg} / \mathrm{kg}$ cocaine followed by retraction of the taking lever, extinction of the house light and illumination of the cue light above the taking lever). A time-out of $30 \mathrm{~s}$ (TO30) was imposed after every infusion, after which another infusion cycle started with the presentation of the drug seeking lever. Thus, animals self-administered cocaine under an RI2/FR1:TO30 chained cocaine seeking/taking schedule. Each daily session lasted $3 \mathrm{~h}$ or 12 infusions, whichever happened first. The RI and TO components of the chained schedule were then increased: RI2/FR1:TO30-RI20/FR1: TO120-RI60/FR1:TO300-RI120/FR1:TO600. Animals received at least 2 daily sessions on each schedule and were allowed at least 6 sessions on the final RI120/FR1:TO600 schedule. Sucrose was simultaneously available on the RI60 schedule throughout the experiment. After stable responding on the final chained schedule was achieved, drug taking was devalued by daily $2 \mathrm{~h}$ extinction sessions. In these sessions only the drug taking lever was present and lever responding had no consequences. Sucrose was still available. After 13 drug taking extinction sessions, drug seeking was assessed in a 5 min test. During this test only the drug seeking lever was present and responses had no consequences. Drug taking was then revalued in two sessions in which animals were allowed to earn cocaine infusions under an FR1 schedule (40 infusions or $2 \mathrm{~h}$, whichever came first) with only the drug taking lever present. A second 5 min drug seeking test was then administered. Thus, cocaine seeking was assessed in two identical tests, after devaluation and again after revaluation of the outcome of the cocaine seeking lever (the drug taking lever).

Experiment 2. The protocol was identical to that in experiment 1 except that after completing the cocaine seeking test, an additional 36 sessions (RI120/FR1:TO600; 3 sessions/d $\times 12$ d) in which rats received 12 infusions/session were conducted. Each of the three daily sessions was separated by $120 \mathrm{~min}$ and occurred during the light phase of the reverse light/dark cycle. Thereafter, extinction of the drug taking lever followed by cocaine seeking tests under devalued and revalued conditions was performed exactly as described for initial training. In this way, the effects of devaluation of the drug taking lever on drug seeking were evaluated in an identical manner both after initial limited training and after extensive drug experience.

Experiment 3. The protocol was identical to that of experiment 2, with the exception that no extinction and drug seeking tests were given after short training. Animals transitioned to the extended training protocol immediately after stable baseline responding was achieved for 6 sessions. Extended training, which occurred during the light phase of the light/ dark cycle, consisted of 30 sessions (3 sessions/d, 2 h apart) and was followed by extinction of the taking lever and the corresponding cocaine seeking tests. The dorsolateral striatum was transiently inactivated in half of the animals by microinjecting $1 \mu \mathrm{l}$ of $4 \%$ lidocaine bilaterally $5 \mathrm{~min}$ before both cocaine seeking tests (e.g., devalued and revalued conditions). Control animals received an equivalent volume of aCSF. For microinjections, a microinjection cannula (C315I, Plastics One) which extended $1 \mathrm{~mm}$ below the tip of the guide was inserted into the guide cannula. One microliter of solution was infused over $1 \mathrm{~min}$. The microinjection cannula was removed 2 min later to allow for diffusion of the solution into the tissue.

\section{Histology}

At the end of experiment 3 , cresyl violet $(1 \% \times 1 \mu \mathrm{l})$ was bilaterally microinjected into the guide cannulae. The animals were then killed and brains removed and frozen on dry ice. Histological verification of the microinjection site was performed in $30 \mu \mathrm{m}$ frozen sections.

\section{Data analysis}

Statistical analysis was conducted using a paired $t$ test to compare cocaine-seeking responses under devalued and revalued conditions (experiment 1). A two-way repeated-measures ANOVA with value of the 

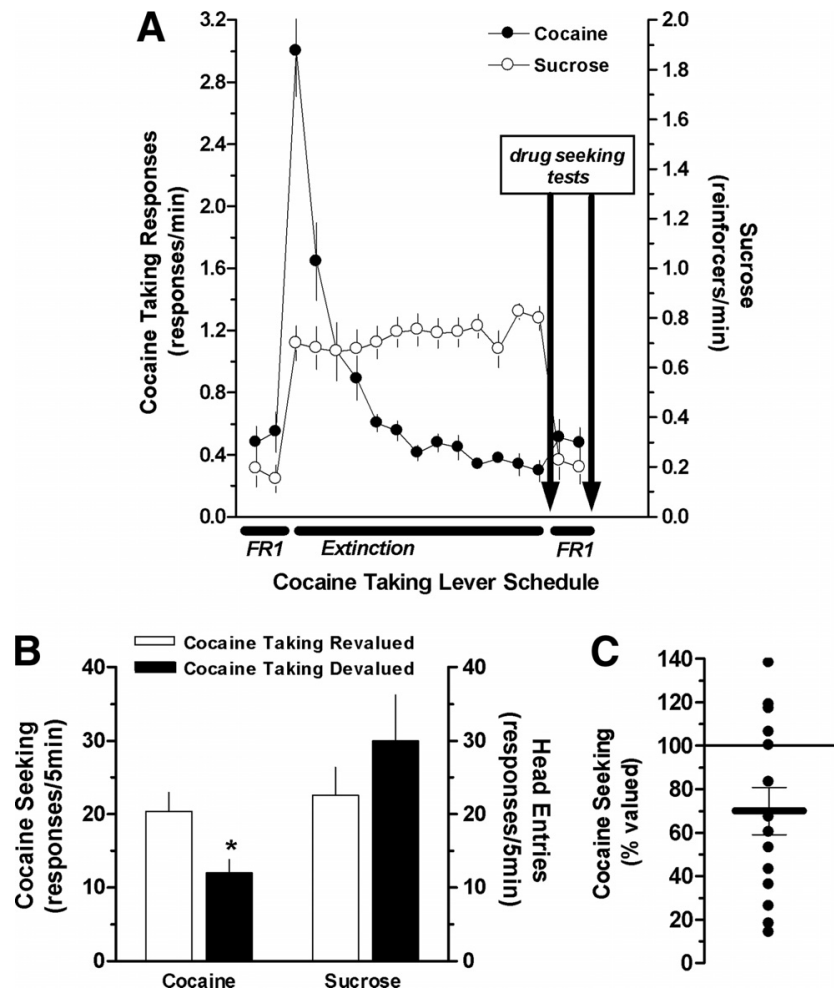

Figure 1. A, Devaluation testing: experiment 1. After stable acquisition of the drug seeking/ taking chained schedule, the cocaine taking link was devalued by extinction. Responses on the drug seeking lever and head entries into the sucrose magazine were measured under extinction conditions during 5 min tests before and after revaluation of the drug taking link (arrows) by two sessions where cocaine infusions were available again under an FR1 schedule. $\boldsymbol{B}$, Cocaine seeking responses and head entries into the sucrose magazine during tests conducted under devalued and revalued conditions. C, The magnitude of the devaluation effect was calculated as cocaine seeking responses after devaluation expressed as a percentage of seeking responses under the revalued condition for each animal (filled circles, $n=14$ ). Percentage of seeking responses under the revalued condition is plotted for each animal. The average \pm SEM for the group (solid horizontal line) is also shown. All data are expressed as the mean \pm SEM. ${ }^{*} p<$ 0.05 , paired $t$ test.

cocaine taking response as the within-subject factor and duration of training (experiment 2) or lidocaine treatment (experiment 3 ) as the other main factor was used to assess the effects of training history or striatal inactivation on the devaluation of cocaine-seeking responses.

\section{Results}

Experiment 1

Animals learned the RI120/FR1:TO600 chained schedule in $20-25$ sessions and earned 12 cocaine infusions per session in $<3$ h. Withholding cocaine infusions extinguished responses on the drug taking lever (Fig. 1A). In the absence of cocaine infusions, however, the number of sucrose reinforcers earned increased threefold and remained elevated throughout extinction. Both drug taking responses and the number of sucrose reinforcers earned returned to pre-extinction levels when cocaine access, under an FR1 schedule, was resumed. To determine whether devaluation of the cocaine taking response by extinction affected the cocaine seeking link, responding during the two drug seeking tests (devalued and revalued conditions) were compared (Fig. $1 B)$. A paired $t$ test indicated significantly lower cocaine seeking responses under devalued conditions $(t=2.60$, $\mathrm{df}=13, p=$ 0.022). Devaluation of the cocaine taking link of the chain did not significantly alter head entries in the sucrose magazine, suggesting that the decreased cocaine seeking under devalued conditions was not due to a generalized decrease in activity after prolonged extinction. Cocaine seeking after devaluation of the drug taking chain was $70 \pm 11 \%$ of cocaine seeking under revalued conditions (Fig. 1C). However, examination of each animal's data from the cocaine seeking tests revealed devaluation effects ranging from $14 \%$ to $138 \%$. Of the 14 subjects tested, drug seeking in 8 animals was clearly attenuated by outcome devaluation as evidenced by a $33-86 \%$ decrease in responding. However, responding of 6 animals after devaluation was $>80 \%$ of that under the revalued condition, indicating poor sensitivity to devaluation in these animals. Thus, in this subset of animals ( $43 \%$ of the total), cocaine seeking was not goal-directed but habitual in nature. In experiment 2 we assessed whether the number of animals exhibiting habitual cocaine seeking increased with more extended cocaine seeking experience.

\section{Experiment 2}

To investigate whether extended training would result in development of insensitivity of cocaine seeking to outcome devaluation (i.e., extinction of the cocaine taking link) rats were trained as in experiment 1 but received 36 additional drug seeking/taking sessions. Cocaine and sucrose seeking remained relatively stable throughout the extended training procedure (Fig. 2 A). However, in contrast to experiment 1 , the sensitivity of cocaine seeking to devaluation of the drug taking link was significantly reduced after extended training (Fig. 2 B). Statistical analysis revealed a significant training experience vs devaluation interaction, $F_{(1,5)}=$ $7.659, p=0.04)$. Head entries into the sucrose magazine were not significantly affected by devaluation of cocaine taking regardless of training experience (Fig. $2 C, F_{(1,5)}=1.0, p=0.36$ ), arguing against nonspecific changes in general activity. The percentage change in cocaine seeking for individual animals after devaluation is shown in Figure 2D. Examination of the short training condition data suggest that the significant devaluation effect for the group average, again resulted from a subgroup of animals in which cocaine seeking decreased in excess of $50 \%$ after devaluation (Fig. $2 D$, filled circles). The remaining animals did not show any apparent devaluation effect indicating that responding of these animals was no longer goal-directed (Fig. $2 \mathrm{D}$, open circles). Analysis of both subgroups after additional training revealed a loss of the devaluation effect in the devaluation-sensitive subgroup (Fig. $2 E$, filled circles). In contrast, little change in the performance of the devaluation insensitive subgroup was seen (Fig. 2 E, open circles). These data are consistent with a shift from goal-directed (sensitive to changes in outcome value) to habitual cocaine seeking behavior (insensitive to changes in outcome value) after extended drug seeking experience.

\section{Experiment 3}

Since the dorsolateral striatum is required for habitual behavior (Yin et al., 2004; Yin and Knowlton, 2006), we tested whether transient inactivation of this brain region would block habitual cocaine seeking and reinstate sensitivity to outcome value. After completing the extended training, animals were assigned to two groups with similar cocaine seeking behavior (Fig. $3 A$ ). The drug taking response was then extinguished as in experiments 1 and 2 . However, the dorsolateral striatum was inactivated before each seeking test by local bilateral infusion of $4 \%$ lidocaine. Control animals received aCSF infusions. Three rats were eliminated from the study due to failure to respond under revalued conditions ( 1,3 , and 3 responses, respectively). Two factor repeated-measures ANOVA revealed a significant main effect of devaluation $\left(F_{(1,18)}=26.17, p<0.0001\right)$ and a signifi- 
A
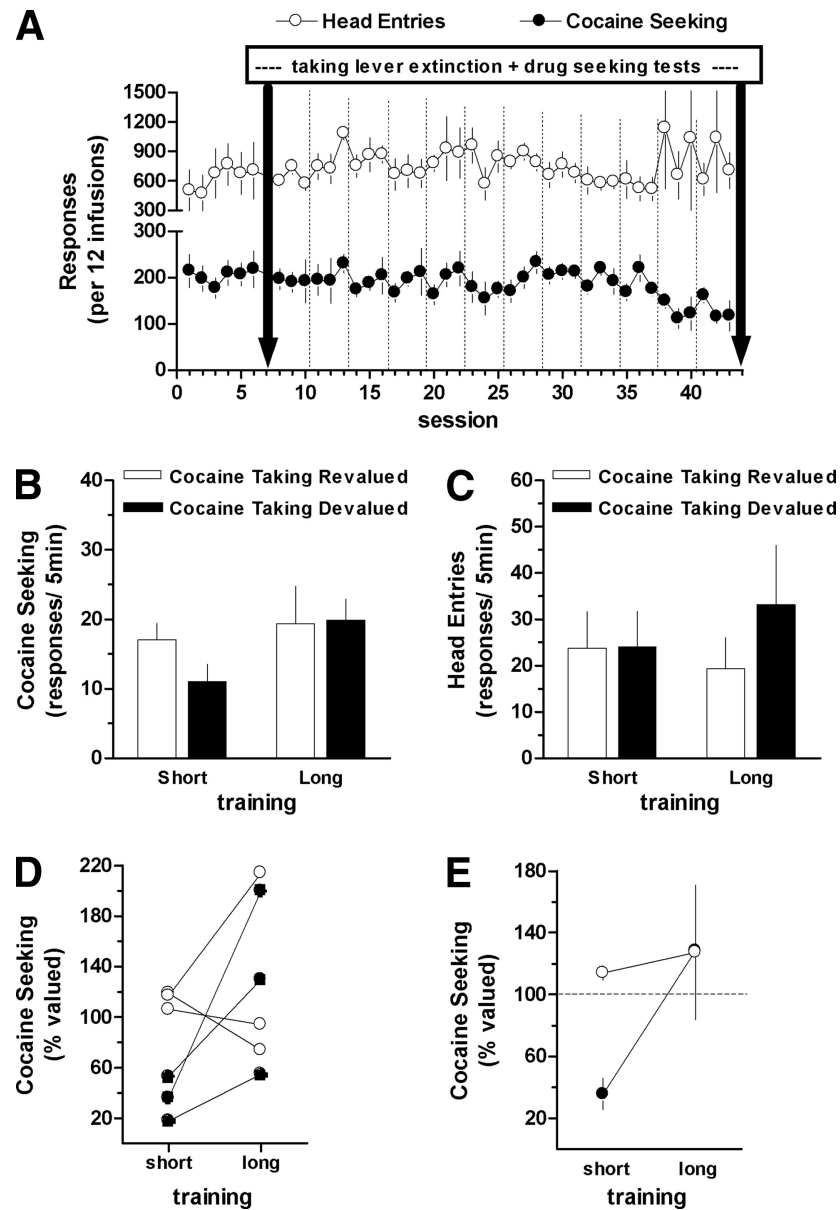

Figure 2. Effects of training duration on sensitivity to devaluation: experiment 2. A, Devaluation tests were performed after short training and again after extended training of the drug seeking/taking chained schedule (arrows). Cocaine seeking responses and head entries into the sucrose magazine for each session, consisting of 12 drug infusions, are shown. Dotted lines indicate each block of 3 daily sessions. The devaluation tests followed the same procedure as in experiment 1. B, Seeking responses for cocaine during the test. $\boldsymbol{C}$, Head entries into the sucrose magazine during the test. $\boldsymbol{D}$, The magnitude of the devaluation effect was calculated as cocaine seeking responses after devaluation expressed as a percentage of seeking responses under the revalued condition for each animal. Animals were assigned to two groups according to their sensitivity to devaluation after short training [3 animals showed no effect of devaluation (open circles) and 3 animals devalued $40 \%$ or more (filled circles)]. $\boldsymbol{E}$, The average \pm SEM devaluation effect for each of these groups is shown. All data are expressed as the mean \pm SEM.

cant infusion $\times$ devaluation interaction $\left(F_{(1,18)}=5.21, p=\right.$ $0.035)$ indicating that the effect of devaluation varied as a function of intrastriatal infusion (Fig. 3B). Post hoc analysis revealed a significant reduction in drug-seeking responses only in rats that had received infusion of lidocaine before testing ( $p<0.01$, Student-Newman-Keuls test). No significant devaluation effect was detected in response to aCSF infusion ( $p>0.05$, Student-Newman-Keuls test), consistent with habitual responding in this group. Head entries in the sucrose magazine were not affected by devaluation of the cocaine taking link (Fig. $3 C, F_{(1,18)}=2.11, p=$ $0.16)$. There was a trend toward increased generalized responding for sucrose in the lidocaine-treated animals regardless of the value of the cocaine taking link, but this trend was not significant $\left(F_{(1,18)}=3.18, p=0.09\right)$.

\section{Discussion}

It has been proposed that habitual drug seeking develops after extended drug experience and that this contributes to the devel-
A
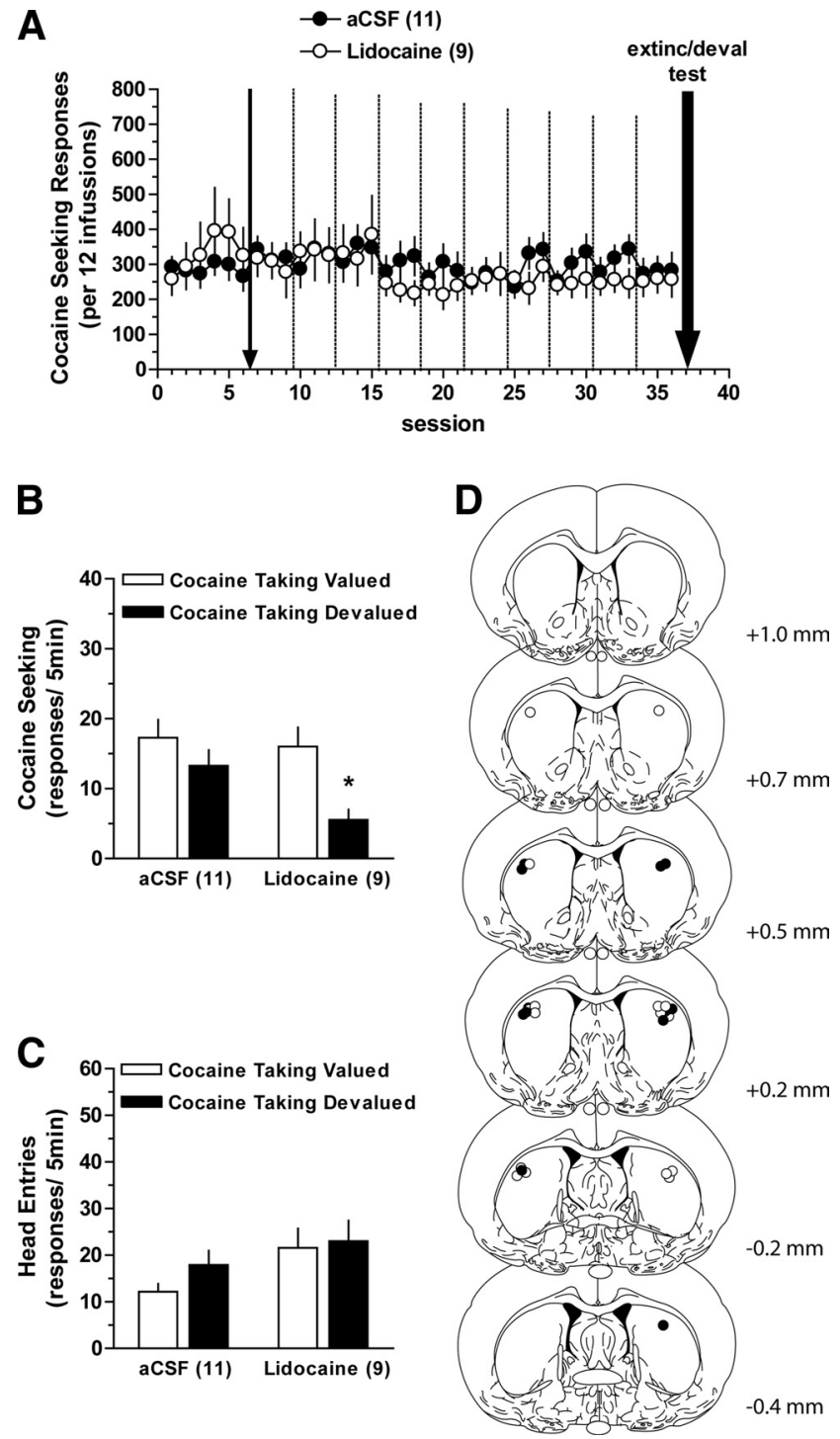

-

Figure 3. Influence of transient inactivation of the dorsolateral striatum on devaluation: experiment 3 . Devaluation testing was performed after an extended training procedure identical to that used in experiment 2 . Animals were divided into 2 groups with similar performance during the drug seeking/taking schedule. $A$, Cocaine-seeking responses for each session, consisting of 12 drug infusions, are shown. Dotted lines separate each block of 3 daily sessions. The devaluation tests took place only at the end of the extended training (arrow) and followed the same procedure as in experiment 1, except that animals received infusions of aCSF or $4 \%$ lidocaine in the dorsolateral striatum immediately before the drug seeking tests. $\boldsymbol{B}_{\text {, Cocaine }}$ seeking responses during testing. C, Head entries responses during testing. D, Histological reconstruction of cannula placements (open circles, aCSF; filled circles, $4 \%$ lidocaine). All data are mean \pm SEM. Number of animals per group is shown in parenthesis. ${ }^{*} p<0.01$, StudentNewman-Keuls post hoc test.

opment of the compulsive drug seeking that characterizes addiction (Everitt et al., 2008). Using a chained schedule of cocaine seeking and taking, the present study provides direct evidence that after extended — but not limited —intravenous cocaine selfadministration, drug seeking becomes habitual and under the control of stimulus-response associations.

In this study, goal-directed versus habitual drug seeking was tested by devaluing the drug taking link of the chained schedule rather than the unconditioned effects of the drug. This procedure differs from standard reward devaluation techniques for oral reinforcers in which the unconditioned reinforcers are devalued by poisoning or satiation. It has been argued that activities directed 
to the procurement of drug (drug seeking) differ from the more immediate, consummatory responses of drug taking (O'Brien et al., 1998). The distinction between seeking and taking responses is important on theoretical and experimental grounds (Olmstead et al., 2001). Indeed, experimental evidence indicates the involvement of distinct processes in seeking and taking responses (Balleine et al., 1995; Arroyo et al., 1998; Ito et al., 2004). Separation of drug seeking from drug taking in the chained schedule used in the present studies allows investigation of habitual drug seeking maintained by cocaine associated stimuli, which is thought to be critical in human addicts (O'Brien et al., 1998), in the absence of the unconditioned effects of drug on operant responding (Everitt and Robbins, 2000).

Analogous to Olmstead et al. (2001), after a moderate amount of training, drug seeking is an action under control of the response-outcome association. Thus, extinction of the drugtaking link of the chain resulted in a significant decrease in responding on the cocaine-seeking lever. Devaluation tests were conducted once stable performance on the RI120/FR1:TO600 chained scheduled was achieved (e.g., 20-25 sessions). Importantly, the use of the variable interval schedule in the drug seeking link of the chain generated high levels of responding (15-30 seeking responses/infusion earned). As a consequence, animals emitted 200-300 responses per session during the short training condition. Although previous work has shown that variable interval schedules are more effective in promoting the development of habits than commonly used fixed ratio schedules (Dickinson et al., 1983), this amount of operant experience was still insufficient to elicit habitual responding. Such findings are noteworthy in that they suggest that self-administration studies in which continuous reinforcement schedules and limited access conditions are used may not provide sufficient drug seeking experience to engage the habit system. Consistent with this contention, only when the number of training sessions was increased (36 additional sessions) was a shift to habitual drug seeking seen (e.g., responding became insensitive to outcome devaluation).

Accumulating evidence indicates that addiction is a disorder in which casual drug use progresses to habitual and ultimately compulsive drug seeking and taking (Everitt and Robbins, 2005). By enabling differentiation of responding that is goal-directed from that which is habitual, the present studies suggest that use of a chained schedule of intravenous cocaine self-administration provides an effective animal model with which to study the mechanisms involved in the development of drug seeking habits (Koob et al., 2004; Vanderschuren and Everitt, 2004; Zernig et al., 2007).

Devaluation of the cocaine taking link of the chain did not decrease approach responses to the sucrose compartment. It should also be noted that the head entry response is not a "pure" instrumental response and also consists of a Pavlovian approach component. Head entries into a food magazine have been used to control for nonspecific response suppression by punishment in studies using drug seeking/taking schedules of cocaine selfadministration (Pelloux et al., 2007). In the present study, head entries increased during extinction of the drug taking link. This finding is consistent with the observation that suppression of cocaine responding increases responding for food (Negus, 2005). Furthermore, the lack of effect of the devaluation procedure on sucrose approach responses during the seeking test indicates that a decrease in drug seeking responding after devaluation or after intrastriatal lidocaine infusions is not the result of a generalized decrease in motor performance due to habituation, sedation or motor impairment.

Examination of each animal's performance during devaluation tests after the moderate training condition revealed that, although drug seeking was goal-directed for the experimental group as a whole, a subset of animals already displayed habitual responding. At present, it is unclear whether individual variability reflects preexisting differences in the learning strategy used (i.e., some animals rely on stimulus-response associations early in the acquisition of a new instrumental task) or faster transition from goal-directed to habitual behavior in this subset of animals. However, the observation that all animals that display goaldirected behavior after moderate training develop habitual drug seeking with extended training (experiment 2) strongly suggests that drug seeking that is initially goal-directed becomes habitual after extensive drug experience.

It has been suggested that compulsive drug seeking can be characterized as a maladaptive stimulus-response habit in which the ultimate goal of the behavior has been devalued, perhaps through tolerance to the rewarding effects of the drug and that the persisting quality of these habits is central to drug addiction (Everitt and Robbins, 2005; Everitt et al., 2008). Additional studies are needed to determine whether individual differences in the rate of development of habitual drug seeking correlate with the propensity to develop compulsive drug seeking, as has been suggested (Everitt et al., 2008), or how it relates to other traits that are thought to predict the development of an addiction phenotype (Belin et al., 2008, 2009b).

Stimulus-response learning has been shown to be dependent on dorsolateral striatal function (Packard et al., 1989; Kantak et al., 2001). To determine whether the dorsolateral striatum is critical for the expression of habitual cocaine-seeking, behavior, lidocaine was used to transiently inactivate this region. In contrast to control animals who exhibited insensitivity to outcome devaluation after extended training, devaluation significantly reduced cocaine seeking in lidocaine-treated rats. Lidocaine has been used extensively to transiently inactivate the dorsal striatum and other brain regions (Van Golf Racht-Delatour and Massioui, 2000; Chang and Gold, 2004; Espina-Marchant et al., 2009). Although, lidocaine may have affected fibers of passage or areas adjacent to the injection site, the present results strongly suggest that disruption of the habit system by transient inactivation of the dorsolateral striatum caused established habitual drug seeking behavior to revert back under control of the goal-directed system. This observation is consistent with imaging studies showing a progressive engagement of the dorsal striatum only after extended drug history in monkeys (Porrino et al., 2004) and humans (Volkow et al., 2006) and with pharmacological studies showing an involvement of the dorsal striatum after prolonged training using second order schedules in rats (Vanderschuren et al., 2005; Fuchs et al., 2006; Belin and Everitt, 2008). These results extend those of Yin et al. (2004), who demonstrated that dorsolateral striatal lesions prevent the development of habitual responding for food reinforcers, and those of Hitchcott and colleagues (2007), who showed that in rats trained to express habitual responding for food, infusions of dopamine into the ventromedial prefrontal cortex restored goal-directed responding. Furthermore, they are consistent with the hypothesis that both, the habit and the goaldirected systems, function in parallel to control instrumental behavior and that disruption of the habit system reengages goaldirected processes that control behavior.

It has been argued that instrumental training engages two independent learning processes controlled respectively by actionoutcome and stimulus-response associations, which operate concurrently (Dickinson, 1985). One important issue is whether the insensitivity to outcome devaluation seen after extended cocaine experience reflects strengthening of the control of behavior 
by the stimulus-response associations or, on the contrary, a disruption of the ability of response-outcome associations to guide behavior. In this regard, Schoenbaum and Setlow have suggested that chronic cocaine experience impairs the ability of response-outcome associations to guide behavior (Schoenbaum and Setlow, 2005). However, transient lidocaine inactivation of the dorsolateral striatum restores sensitivity to outcome value in cocaine experienced animals suggesting that the ability of the response-outcome association to control drug seeking remains intact after extended cocaine experience but is masked or inhibited by the habit system. In this regard, however, it should be noted that Schoenbaum and Setlow (2005) examined Pavlovian rather than instrumental responses which are known to be controlled by different brain systems (Belin et al., 2009a). Moreover, cocaine experience was limited and was experimenter-administered. These differences preclude a direct comparison between studies. However, the present finding that inhibition of the habit system can shift control of drug seeking to a goal-directed, voluntary process, has important implications for medications development given the proposed contribution of habitual behavior to the automaticity of drug seeking observed in addicts as well as to drug-craving and relapse to addiction (Tiffany, 1990).

In summary, after prolonged drug experience, intravenous cocaine seeking becomes a habit insensitive to changes in outcome value. Inactivation of the dorsolateral striatum causes established habitual cocaine seeking to revert back to control of the goal-directed system, suggesting that drug seeking habits may be reversible. The present studies suggest that engagement of the habit system requires more prolonged drug seeking experience than that afforded by most animal drug self-administration studies and are consistent with the notion that different brain systems are engaged as drug self-administration progresses from controlled drug use to abuse and to addiction. Finally, our studies suggest that the devaluation of the drug-taking link of a chained schedule of cocaine selfadministration provides an effective model with which to study the neural substrates underlying goal-directed versus habitual drug seeking.

\section{References}

Adams CD, Dickinson A (1981) Instrumental responding following reinforcer devaluation. Q J Exp Psychol 33:109-121.

Arroyo M, Markou A, Robbins TW, Everitt BJ (1998) Acquisition, maintenance and reinstatement of intravenous cocaine self-administration under a second-order schedule of reinforcement in rats: effects of conditioned cues and continuous access to cocaine. Psychopharmacology (Berl) 140:331-344.

Balleine BW, Dickinson A (1998) Goal-directed instrumental action: contingency and incentive learning and their cortical substrates. Neuropharmacology 37:407-419.

Balleine BW, Garner C, Gonzalez F, Dickinson A (1995) Motivational control of heterogeneous instrumental chains. J Exp Psychol 21:203-217.

Belin D, Everitt BJ (2008) Cocaine seeking habits depend upon dopaminedependent serial connectivity linking the ventral with the dorsal striatum. Neuron 57:432-441.

Belin D, Mar AC, Dalley JW, Robbins TW, Everitt BJ (2008) High impulsivity predicts the switch to compulsive cocaine-taking. Science 320:1352-1355.

Belin D, Jonkman S, Dickinson A, Robbins TW, Everitt BJ (2009a) Parallel and interactive learning processes within the basal ganglia: relevance for the understanding of addiction. Behav Brain Res 199:89-102.

Belin D, Balado E, Piazza PV, Deroche-Gamonet V (2009b) Pattern of intake and drug craving predict the development of cocaine addiction-like behavior in rats. Biol Psychiatry 65:863-868.

Chang Q, Gold PE (2004) Inactivation of dorsolateral striatum impairs ac- quisition of response learning in cue-deficient, but not cue-available, conditions. Behav Neurosci 118:383-388.

Colwill RM, Rescorla RA (1985) Postconditioning devaluation of a reinforcer affects instrumental responding. J Exp Psychol Anim Behav Process 11:120-132.

Corbit LH, Balleine BW (2005) Double dissociation of basolateral and central amygdala lesions on the general and outcome-specific forms of pavlovian-instrumental transfer. J Neurosci 25:962-970.

Dickinson A (1985) Actions and habits: the development of behavioural autonomy. Philos Trans R Soc Lond Biol 308:67-78.

Dickinson A, Nicholas DJ, Adams CD (1983) The effect of the instrumental training contingency on susceptibility to reinforcer devaluation. Q J Exp Psychol B 35:35-51.

Dickinson A, Wood N, Smith JW (2002) Alcohol seeking by rats: action or habit? Q J Exp Psychol B 55:331-348.

Espina-Marchant P, Pinto-Hamuy T, Bustamante D, Morales P, HerreraMarschitz M (2009) Rat strain influences the use of egocentric learning strategies mediated by neostriatum. Exp Brain Res 193:205-212.

Everitt BJ, Robbins TW (2000) Second-order schedules of drug reinforcement in rats and monkeys: measurement of reinforcing efficacy and drug-seeking behaviour. Psychopharmacology (Berl) 153:17-30.

Everitt BJ, Robbins TW (2005) Neural systems of reinforcement for drug addiction: from actions to habits to compulsion. Nat Neurosci 8:1481-1489.

Everitt BJ, Belin D, Economidou D, Pelloux Y, Dalley JW, Robbins TW (2008) Review. Neural mechanisms underlying the vulnerability to develop compulsive drug-seeking habits and addiction. Philos Trans R Soc Lond B Biol Sci 363:3125-3135.

Faure A, Haberland U, Condé F, El Massioui N (2005) Lesion to the nigrostriatal dopamine system disrupts stimulus-response habit formation. J Neurosci 25:2771-2780.

Fuchs RA, Branham RK, See RE (2006) Different neural substrates mediate cocaine seeking after abstinence versus extinction training: a critical role for the dorsolateral caudate-putamen. J Neurosci 26:3584-3588.

Hitchcott PK, Quinn JJ, Taylor JR (2007) Bidirectional modulation of goaldirected actions by prefrontal cortical dopamine. Cereb Cortex 17:2820-2827.

Ito R, Dalley JW, Robbins TW, Everitt BJ (2002) Dopamine release in the dorsal striatum during cocaine-seeking behavior under the control of a drug-associated cue. J Neurosci 22:6247-6253.

Ito R, Robbins TW, Everitt BJ (2004) Differential control over cocaineseeking behavior by nucleus accumbens core and shell. Nat Neurosci 7:389-397.

Kantak KM, Green-Jordan K, Valencia E, Kremin T, Eichenbaum HB (2001) Cognitive task performance after lidocaine-induced inactivation of different sites within the basolateral amygdala and dorsal striatum. Behav Neurosci 115:589-601.

Koob GF, Ahmed SH, Boutrel B, Chen SA, Kenny PJ, Markou A, O’Dell LE, Parsons LH, Sanna PP (2004) Neurobiological mechanisms in the transition from drug use to drug dependence. Neurosci Biobehav Rev 27:739-749.

Miles FJ, Everitt BJ, Dickinson A (2003) Oral cocaine seeking by rats: action or habit? Behav Neurosci 117:927-938.

Negus SS (2005) Effects of punishment on choice between cocaine and food in rhesus monkeys. Psychopharmacology (Berl) 181:244-252.

Nelson A, Killcross S (2006) Amphetamine exposure enhances habit formation. J Neurosci 26:3805-3812.

Nordquist RE, Voorn P, de Mooij-van Malsen JG, Joosten RN, Pennartz CM, Vanderschuren LJ (2007) Augmented reinforcer value and accelerated habit formation after repeated amphetamine treatment. Eur Neuropsychopharmacol 17:532-540.

O’Brien CP, Childress AR, Ehrman R, Robbins SJ (1998) Conditioning factors in drug abuse: can they explain compulsion? J Psychopharmacol 12:15-22.

Olmstead MC, Lafond MV, Everitt BJ, Dickinson A (2001) Cocaine seeking by rats is a goal-directed action. Behav Neurosci 115:394-402.

Packard MG, Knowlton BJ (2002) Learning and memory functions of the basal ganglia. Annu Rev Neurosci 25:563-593.

Packard MG, Hirsh R, White NM (1989) Differential effects of fornix and caudate nucleus lesions on two radial maze tasks: evidence for multiple memory systems. J Neurosci 9:1465-1472.

Pelloux Y, Everitt BJ, Dickinson A (2007) Compulsive drug seeking by rats under punishment: effects of drug taking history. Psychopharmacology (Berl) 194:127-137. 
Porrino LJ, Lyons D, Smith HR, Daunais JB, Nader MA (2004) Cocaine self-administration produces a progressive involvement of limbic, association, and sensorimotor striatal domains. J Neurosci 24:3554-3562.

Schoenbaum G, Setlow B (2005) Cocaine makes actions insensitive to outcomes but not extinction: implications for altered orbitofrontalamygdalar function. Cereb Cortex 15:1162-1169.

Tiffany ST (1990) A cognitive model of drug urges and drug-use behavior: role of automatic and nonautomatic processes. Psychol Rev 97:147-168.

Vanderschuren LJ, Everitt BJ (2004) Drug seeking becomes compulsive after prolonged cocaine self-administration. Science 305:1017-1019.

Vanderschuren LJ, Di Ciano P, Everitt BJ (2005) Involvement of the dorsal striatum in cue-controlled cocaine seeking. J Neurosci 25:8665-8670.

Van Golf Racht-Delatour B, Massioui NE (2000) Alleviation of overtraining reversal effect by transient inactivation of the dorsal striatum. Eur J Neurosci 12:3343-3350.
Volkow ND, Wang GJ, Telang F, Fowler JS, Logan J, Childress AR, Jayne M, Ma Y, Wong C (2006) Cocaine cues and dopamine in dorsal striatum: mechanism of craving in cocaine addiction. J Neurosci 26:6583-6588.

White NM (1996) Addictive drugs as reinforcers: multiple partial actions on memory systems. Addiction 91:921-949.

Yin HH, Knowlton BJ (2006) The role of the basal ganglia in habit formation. Nat Rev Neurosci 7:464-476.

Yin HH, Knowlton BJ, Balleine BW (2004) Lesions of dorsolateral striatum preserve outcome expectancy but disrupt habit formation in instrumental learning. Eur J Neurosci 19:181-189.

Zernig G, Ahmed SH, Cardinal RN, Morgan D, Acquas E, Foltin RW, Vezina P, Negus SS, Crespo JA, Stöckl P, Grubinger P, Madlung E, Haring C, Kurz M, Saria A (2007) Explaining the escalation of drug use in substance dependence: models and appropriate animal laboratory tests. Pharmacology 80:65-119. 